\title{
Effectiveness of Task Sharing and Task Shifting on the Uptake of Family Planning in Kenya
}

\author{
Leopold Ouedraogo ${ }^{1}$, Okech Mollent ${ }^{2 *}$, Gondi Joel ${ }^{3}$ \\ ${ }^{1}$ World Health Organization, Brazzaville, Congo \\ ${ }^{2}$ Peak Moments Global Health Solutions, Nairobi, Kenya \\ ${ }^{3}$ Ministry of Health, Nairobi, Kenya \\ Email: *mollentakinyi@gmail.com
}

How to cite this paper: Ouedraogo, L. Mollent, O. and Joel, G. (2020) Effectiveness of Task Sharing and Task Shifting on the Uptake of Family Planning in Kenya. $A d$ vances in Reproductive Sciences, 8, 209-220. https://doi.org/10.4236/arsci.2020.84018

Received: August 22, 2020

Accepted: October 20, 2020

Published: October 23, 2020

Copyright (c) 2020 by author(s) and Scientific Research Publishing Inc. This work is licensed under the Creative Commons Attribution International License (CC BY 4.0).

http://creativecommons.org/licenses/by/4.0/

\section{(cc) (i) Open Access}

\begin{abstract}
Background: Contraception is an inexpensive and cost-effective intervention, but health workforce shortages and restrictive policies on the roles of mid and lower-level cadres limit access to effective contraceptive methods in many settings. Task sharing and task shifting are strategies that can be adopted to increase uptake of health services including family planning. Methods: We collected data through online survey, key informant interviews and focused grouped discussions with an intervention group and that implemented the task sharing and task shifting policy guidelines and a control group that did not implement the policy. A total of 434 questionnaires were filled by health workers' in primary health care facilities to assess effectiveness of task sharing and task shifting on the uptake of family planning services including its strengths and challenges. The questionnaire was designed with the aim of getting data on services provided by the cadres on effectiveness (number of clients, increase in use of methods, access to services), how they perceive these tasks, the bottlenecks and facilitating factors associated with the practice of task sharing and task shifting. Results: We found out that the task sharing and task shifting can expand and increase access to services as stated by $95 \%$ of the respondents. Most community health workers provided more of the family planning services at $45 \%$ with only $5 \%$ of the services of family planning being provided by medical officers. $98 \%$ of family planning services were integrated with other services. Task shifting was beneficial to the health care providers as well as the clients and the success of task sharing and task shifting depended on training, supportive supervision and a regulated environment through policies. Conclusion: The study shows that formalized task sharing and task shifting can increase health service uptake especially when community health workers are involved to provide services in the community. This leads to increased service provision, equivalent health professional performance across cadres and patient outcomes in the provision of family
\end{abstract}


planning services.

\section{Keywords}

Task Sharing, Task Shifting, Family Planning, Uptake, Effectiveness

\section{Background}

Increasing family planning (FP) uptake and providing universal access to reproductive health in resource poor settings has been severely constrained by a shortage of health workers required to deliver interventions [1]. The World Health Organization (WHO) in the Global Health Statistics Report of 2018 projects a shortfall of 12.9 million health workers by 2035 , mostly in low- and lower-middle income countries and recommends a threshold of 34.5 skilled health professionals (midwives, nurses and physicians) per 10,000 population [2]. The shortage is most severe in the poorest countries, especially in sub-Saharan Africa, where health workers are most needed. Kenya like other countries faces strains in meeting demands for provision of health services because of limited human resources for health (HRH). This situation has been exacerbated by the continued "brain drain" of highly trained medical workers and more recently by the increased demand for services [3]. In a health workforce assessment conducted in 2016, Kenya had a total of 31, 412 employed in both public and private sector and classified 13 counties as having critical HRH shortages [4]. These numbers were far much below the required numbers at 138,266 as proposed in the staffing norms and standards [5]. In order to increase access to health services, the country developed a Task Sharing and Task Shifting Policy Guidelines on 2017 to expand roles of existing workforce while also acknowledging the role that can be played by community health workers in increasing access to health services [6] [7]. Currently, Kenyan health workforce density stands at 9.81 health workers per 10,000 population and indeed will take several years to achieve the WHO recommended minimum HRH levels [8]. Task shifting involves rationally moving some tasks from one cadre to another, such as nurses, midwives, or community health workers. Tasks are moved where appropriate to less specialized health workers. By reorganizing the workforce in this way can make more efficient use of the human resources currently available [9], for example, when doctors are in short supply, a qualified nurse could often prescribe and dispense medication. Some informal task sharing and shifting had been going in the country un documented and the development of the TSTSPG would help in reorganizing health service delivery through formalized task sharing and task shifting especially where shortages of HRH are so high to improve intended health outcomes as outlined in the Kenya Health Policy and Vision 2030 [10].

Contraception is an inexpensive and cost-effective intervention [11], but health workforce shortages and restrictive policies on the roles of mid and low- 
er-level cadres limit access to effective contraceptive methods in many settings [12]. The growing use of contraceptive methods in recent decades has resulted not only in improvements in health-related outcomes such as reduced unintended pregnancies, high-risk pregnancies, maternal mortality, and infant mortality, but also in improvements in schooling and economic outcomes, especially for girls and women [13]. Beyond the impacts of contraceptive use at the individual level, there are benefits at the population level. From a macroeconomic perspective, reductions in fertility enhance economic growth as a result of reduced youth dependency and an increased number of women participating in paid labour [14]. Continued rapid population growth presents challenges for achieving the 2030 Agenda for Sustainable Development, particularly in sub-Saharan Africa where countries must provide health-care services. In family planning, perhaps the most important reason for task sharing and shifting is to bring services to women with poor access [15].

Kenya recognizes that the provision of family planning services as an essential human right that enables women to prevent unwanted pregnancies and control family sizes. The $\mathrm{MoH}$ has thus committed to improving uptake family planning (FP) services and especially increase national modern contraceptive prevalence rate (mCPR) from $56 \%$ to $58 \%$ in 2020 and to $70 \%$ by 2030 . The country further aims at increasing mCPR from $61 \%$ to $66 \%$ by the year 2030 as documented in the Kenya Family Planning Costed Implementation Plan of 2017-2020 [16].

Historically, most health service interventions including family planning have been offered in clinical settings by mainstream health workers in Kenya. The high fertility rates and huge unmet needs for family planning in the country have however inspired the rethink of this approach to help broaden access to contraception. Long distances that clients have to walk to clinics, long waits at the clinics for services as a consequence of shortage of skilled health workers, the frequent industrial actions that have disrupted services from time to time and the occasional stock outs of commodities have made it difficult for women desiring to use contraceptives to get them in a timely manner [17] [18]. Acknowledging further gaps in skills, inequity in distribution regionally and at facility levels, the country realized the need for greatly involving the community health workers to include multiple providers as an opportunity to expand and improve access to family planning as enumerated in the TSTSPG and the Community Health Strategy [6] [7].

Through a MoH Circular, thirteen counties with critical HRH shortages were authorized to utilize community health workers (CHWs) to provide family planning services to include depomedroxyprogesterone acetate-Intramuscular (DMPA-IM) and depomedroxyprogesterone acetate-sub cutaneous (DMPA-SC) alongside the condoms and pills in the rural and marginalized areas to increase family planning access. It is against this background that the study sought to determine effectiveness of implementing task sharing and task shifting on the uptake of family planning in selected counties of Kenya. 
We set out to assess the effectiveness of task sharing and task shifting on the uptake of family planning selected counties of Kenya. We categorized the counties into two categories; those counties that had authority to implement the Task Sharing and Task Shifting Policy and Guidelines (TSTSPG) of 2017 and those that were not authorized to implement the TSTSPG (2017). Our study aims to add evidence on strategies that increase and optimize available health workers in cases of shortages and provide evidence on the effectiveness of task sharing and task shifting on family planning uptake especially where legal frameworks through policies and policy guidelines exist.

\section{Methods}

\section{Study Design}

The research adopted both qualitative and quantitative methods. While the initial intention of the study was to adopt a quasi-randomized control trial to evaluate the effectiveness of task sharing and task shifting, this was not possible as the control sites had implemented the interventions as well. Therefore a descriptive cross-sectional research design involving different health professionals drawnfrom two sets of counties ( 5 of those that were authorized to implement the TSTSPG-(Turkana, Kajiado, Baringo, Narok and Samburu) and those that were not authorized to implement the TSTSPG-(Kisumu, Nandi, Siaya, Migori and Homabay) through online survey, key informant interviews and focused group discussions using designed and pretested tools. We focused on understanding task sharing and tasks shifting in Kenya, the implementation structures, the cadres involved and their tasks, to what extent task sharing and task shifting was successful, bottlenecks and facilitators of task sharing and task shifting.

\section{Respondents}

Through simple random sampling, respondents were drawn from healthcare providers-medical officers, nurses, clinical officers and community health workers at the community units, dispensaries, health centers and hospitals offering family planning services and beneficiaries of the services. A total of 25 functioning community units, 18 dispensaries, 17 health centres and 11 hospitals included and the family planning services provided in the facilities from 2017 to 2019 was included in the analysis. Since the population of health workers offering family planning services in the 10 counties under study was less than 10,000, the researcher used a sample size calculator (Qualtrics, 2019). An assumption of $95 \%$ confidence level and a margin error of $5 \%$ giving a sample size of 367 . Those who responded were more than the sample by 67 giving a total of 434 health workers. Five national county directors at $\mathrm{MoH}$ and 10 county reproductive health coordinators from the 10 counties included in the study.

\section{Data collection}

The researchers designed and pre tested both closed and open ended questionnaires that were administered online through a Survey Monkey to 424 health 
care workers through the CRHC from $13^{\text {th }}$ May to $23^{\text {rd }}$ May 2020. Secondary data was obtained through a review of family planning reports, publications, customer feedbacks and data sets of FP services from the District Health Information Systems2 (DHIS2). These provided indicators of effectiveness of task sharing and task shifting on the uptake of family planning services. Telephone interviews were conducted with the ten coordinators and clients while an FGD with 5 national directors of reproductive health were conducted.

\section{Data analysis}

Notes from KII interviews were transcribed and then coded into thematic areas and presented through frequencies, tables and graphs and described in a narrarive form on the main characteristics of the variables under study. Quantitative data was processed and analyzed using Microsoft Excel 2013.

\section{Ethical considerations}

This study was approved by the Research and Ethics committee of National Commission of Science and Technology and Innovations (NACOSTI), MoH and County Health Departments (CHDs) in Kenya. Written consent was obtained from participants. All participants who consented to take part in the survey were well informed about the purpose of the study and why they were being asked to participate. They were also informed that participation was voluntary and they had the right to withdraw from the study at any time of the study even after they had participated. We also assured the respondents of their confidentiality, anonymity, and privacy. We also requested for consent for the audio recordings of the focus group discussions with the $\mathrm{MoH}$ directors.

\section{Results}

A total of 424 healthcare providers responded by filling in all the questions giving a response rate of $118.3 \%$ against the proposed sample of 367 . The response rate in this study surpassed the target. The responses were obtained from 10 counties as shown in Figure 1 and Figure 2 showing the distribution of the participants to either the control group $(n=77)$ or the intervention group $(n=$ 347). Majority of the respondents (37\%) were aged between 35 and 44 years old. Most of the respondents had attained college level of education (43\%) and most of them were nurses (45\%).

Out of the 424 respondents from the intervention and control groups, 411 (95\%) recorded that they were aware of the policy and its role, $12(3 \%)$ were not sure of such policy guidelines' existence and the role they played in the optimization of the staffing numbers. $6(1 \%)$ did not know whether such a policy existed and only $5(1 \%)$ stated that they were not aware of the policy guidelines on task sharing and task shifting in the health sector. The health care providers at all the levels of care registered broad consensus regarding their awareness of the implementation of task sharing and task shifting practices in the country. In relation to this common understanding among informants, they noted the importance of 


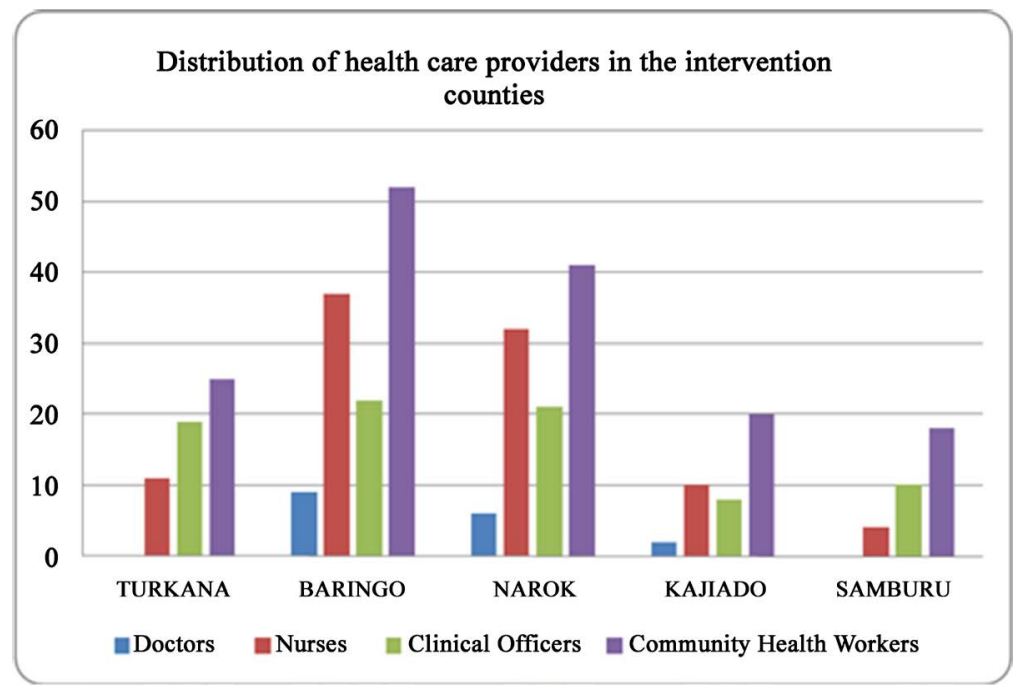

Figure 1. Distribution of respondents in the intervention counties.

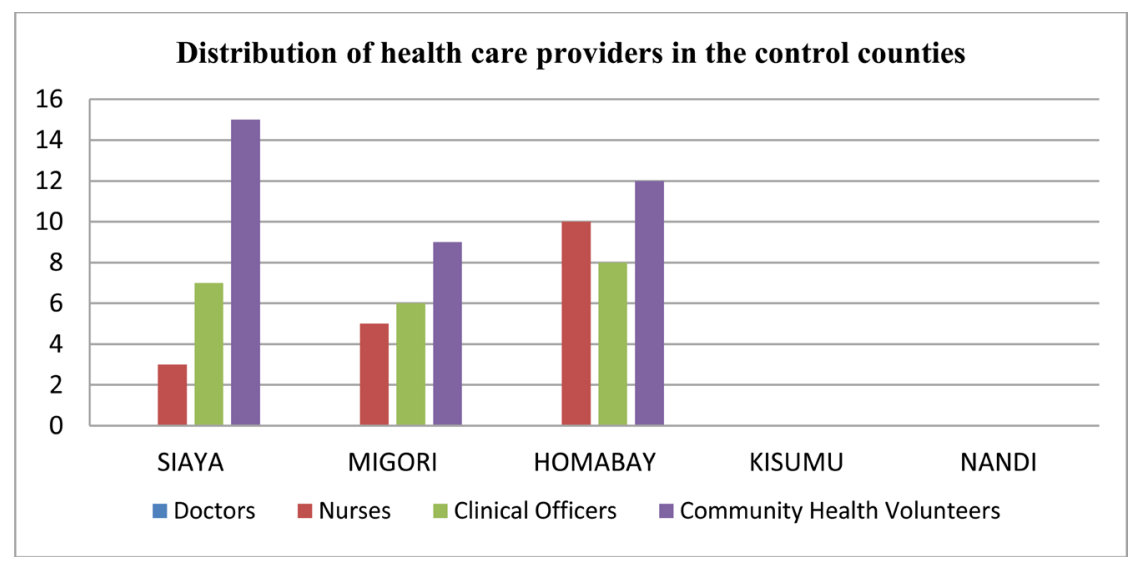

Figure 2. Distribution of respondents in the control counties.

the policy guidelines that mandated them to offer particular services.

On effectiveness of task sharing and task shifting through engagement of the CHWs, the study noted that the mainly sought services were short term family planning methods and the preferred providers were the community health workers at $43 \%$. The nurses only provided short terms services to $26 \%$ of the clients, $24 \%$ by clinical officers $24 \%$ and only $5 \%$ sought the services from medical officers. Its integration with other services like postpartum services (100\%), post-abortion care (92\%) and HIV services (78\%).

Out of the 133 clients interviewed, 80(60\%) preferred the short term acting methods of family planning from the options available in the facilities, followed by $29 \%$ (39/133) of clients who preferred long acting methods while $9(7 \%)$ preferred natural method. The least preferred methods ((4\%) was permanent methods.

Overall, all the groups of the respondents provided benefits of the task sharing and task shifting as shown in Table 1. Majority of healthcare providers (99\%) reported that the benefit of task sharing and task shifting was that it enables a 
Table 1. Benefits of task sharing and task shifting.

\begin{tabular}{|c|c|c|}
\hline & Responses of the health care providers and coordinators & Respondents \% \\
\hline 1 & A wide range of providers enables access and increases uptake of FP services & $99 \%$ \\
\hline 2 & Sharing routine tasks allows higher cadre clinicians more time to use their specialized skills. & $96 \%$ \\
\hline 3 & Reduces workload to the few available staff & $97 \%$ \\
\hline 4 & It helps in enhancing community follow up & $89 \%$ \\
\hline 5 & Ensures greater efficiency with higher productivity from each worker & $31 \%$ \\
\hline \multicolumn{2}{|r|}{ Responses from the directors RHMSU } & $\%$ \\
\hline 1 & The approach has the potential to positively contribute to strengthening health systems & $100 \%$ \\
\hline 2 & The rationale behind the transferring of these tasks is that the alternative would be no service to those in need & $100 \%$ \\
\hline 3 & Allows the facilities to increase access to services and coverage & \\
\hline 4 & Rapidly expands the number of health service providers by better using the available human resources. & $100 \%$ \\
\hline 5 & Presents a viable option for health system cost savings & $100 \%$ \\
\hline 6 & $\begin{array}{l}\text { May be used to train alternate health care workers or lay persons to perform tasks generally } \\
\text { considered to be within the purview of a few }\end{array}$ & $100 \%$ \\
\hline 7 & Specialization promotes quality & $100 \%$ \\
\hline 8 & It encourages staff retention especially in rural areas & $60 \%$ \\
\hline \multicolumn{3}{|c|}{ Responses from the clients } \\
\hline 1 & Lowers per-visit cost since community health workers are flexible & $98 \%$ \\
\hline 2 & Clients are able to choose a preferred care provider. & $75 \%$ \\
\hline 3 & Saves time for going to the facilities some of which are very far away & $100 \%$ \\
\hline
\end{tabular}

wide range of providers to access and increases uptake of FP services, while $100 \%$ of directors at $\mathrm{MoH}$ reported the potential of the approach to positively contribute to strengthening health systems. $98 \%$ of clients reported the approach as having the potential to lower cost per visit since community health volunteers are flexible and would avail themselves without them traveling to the facilities.

On the overall judgment on the effectiveness of task sharing and task shifting as a strategy to increase the uptake on family planning services, (52\%) stated task sharing and task shifting was good, followed by $33 \%$ who stated that task sharing and task shifting as a practice was very good and $9 \%$ judged the strategy as excellent. Only $6 \%$ stated that task sharing and task shifting was average as indicated in the trends of increase in family planning uptake.

Effectiveness of task sharing and task shifting is shown in Figure 3 and Figure 4. Kajiado saw a sharp increase in 2018 compared to 2017 where it recorded 66.1 from $48.9 \%$. The year 2017 had low uptakes in Turkana and Baringo as statistics showed some gaps that were due health a prolonged health worker strike. The health workers stated that sometimes the reduced uptake within the years was due to stock outs of family planning commodities. Figure 3 and Figure 4 provided the uptake of family planning in the intervention counties 2017-2019. 


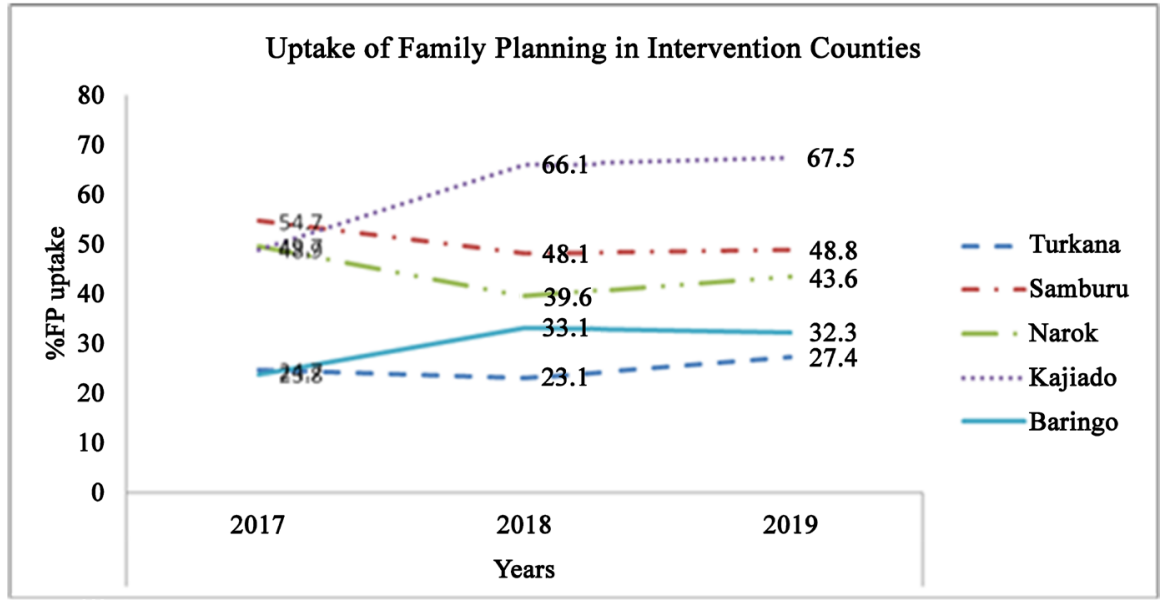

Figure 3. Trends in family planning uptake in the intervention counties 2017-2019.

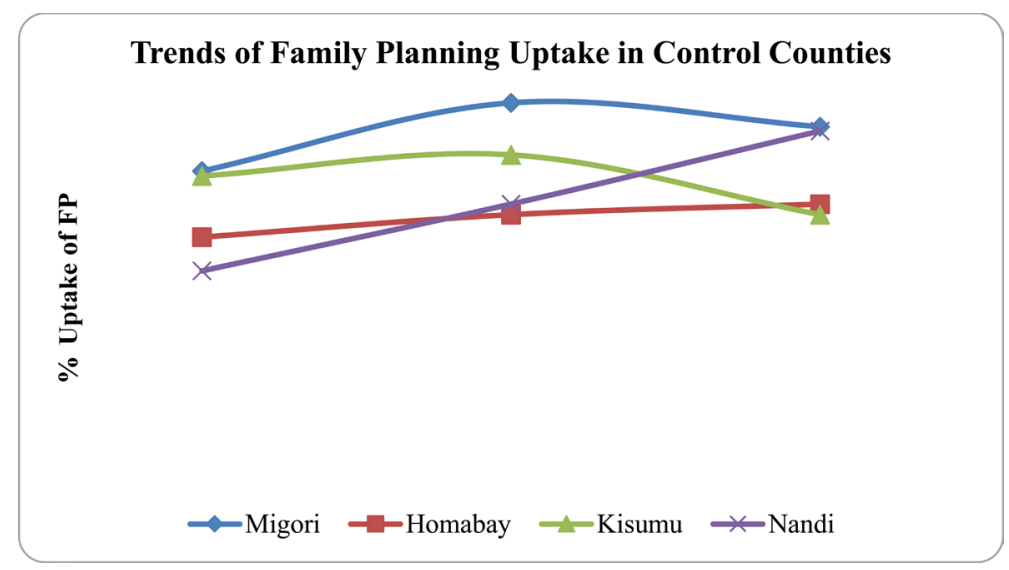

Figure 4. Family Planning trends in the control counties from 2017-2019.

\section{Discussion}

Kenya's health worker shortage hampers delivery of health services and especially in rural areas. The country has committed to increase access to and use of quality FP services by all and especially by the poor, vulnerable and marginalized populations- and achieves a mCPR of $58 \%$ by 2020 and so the TSTSPG is one such strategy under implementation towards that goal.

The findings suggest that acceptance of new roles and inclusion of community health workers has been strengthened by Kenya formalizing the TSTSPG involving regulatory councils, training institutions and other experts. By expanding the range of providers and involving community health care providers an upward trend has been registered with Kajiado County steadily registering increases from 54.7\%, 66.1 and 67.7 respectively from 2017 to 2019 . A study by Kessler [19] revealed that post-implementation of a task sharing policy; health care assistant roles were viewed very positively by nurses, patients and the new cadres themselves. They do however warn that the specific usefulness of these new human resources is rarely well understood, and hence, they are not strategically deployed despite their obvious potential in addressing $\mathrm{HRH}$ needs. 
The expansion of roles of nurses and clinical officers and the introduction of community health care workers in providing family planning services in the dispensaries, health centers and community units has tremendously increased access to the women and girls. From the results, the community health care providers appear to reach out to more clients who are far away from the facilities and they are popular with short acting methods. Health workers serving at clinical settings are the link with CHWs, and they foster local ownership of community-based interventions, ensure sustainability, efficiency, and to improve rapport between facility- and community-based health care providers. Health workers continue to provide mentorship and follow up [20] [21] [22].

Findings indicate that CHWs have often shown convincing effects on program outputs, outcomes, and impacts. A review by [23] concludes that “...there is robust evidence that $\mathrm{CHWs}$ can undertake actions that lead to improved health outcomes..." while a second commentator states "There is no longer any place for discussion of whether $\mathrm{CHW}$ can be key actors in achieving adequate health care" [24]. However, the same authors also concede that many programs are not successful and call for careful planning and research to identify and overcome problems. Task sharing has been applied for many years in family planning programs as various tasks get shared between doctors and nurses or between nurses and community health workers. The consensus reported in a recent WHO document, “Task Shifting to Tackle Health Worker Shortages”, was that task sharing "will positively affect health outcome... Good management, support, supervision and political commitment," are needed for success [25].

To increase family planning uptake in most countries [26] [27] suggest that novel approaches such as integration of family planning with other health services are needed to extend access to family planning services to women and couples who desire to limit or space their childbearing but are not currently using contraceptives.

The study had some limitations. It intended to cover two different sets of counties (the intervention counties and the control counties) in Kenya to compare the results.

1) The counties that had been authorized to implement the TSTSPG due to staffing shortages.

2) The counties that were perceived to have adequate staffing; and thus had restrictions on the use of the TSTSPG.

However, during data collection it was noted that all the counties both implemented the policy due to staffing shortages of one cadre or the other. Therefore, the analysis had no opportunity to separate the intervention counties and the control counties and only recorded the effectiveness of task sharing and shifting on the uptake of family planning in Kenya. Lastly, access to client or beneficiaries of family planning was limited as the targeted numbers could not be reached thus low response. However, the study used the client satisfaction survey reports to fill in the gap. 
The results reveal that task sharing and task shifting are beneficial to both the health care providers and the beneficiaries. To the nurses and clinical officers, task sharing and task shifting freed up their time to undertake more complex tasks while the community healthcare workers undertook counselling and provision of short acting family planning methods.

While challenges like commodities stock outs, professional resistance and inadequate remuneration exist; opportunities exist for improving and scaling up task sharing and task shifting. We recommend further studies that compare task sharing and task shifting where policies exist with where no policies formalizing the process exist. Standardization and harmonization of training and supportive supervision are needed to ensure that the new cadres can effectively perform. The supply of family planning commodities should be addressed together with incentives for the success of the task sharing and task shifting strategy.

\section{Conclusion}

The study concluded that task sharing and task shifting interventions have the potential in increasing family planning uptake especially where the policy environment is conducive. Delivery of services in the community increases access to the communities due to the flexibility of delivery and community health workers play a critical role in community services, thereby reducing unmet family planning needs. However, standardized training, supportive supervision and incentives are critical to ensure sustainability of the strategy.

\section{Authors' Contributions}

All the authors have read and approved the final manuscript.

\section{Conflicts of Interest}

The authors declare no conflicts of interest regarding the publication of this paper.

\section{References}

[1] World Health Organization (2006) The World Health Report 2006: Working Together for Health. WHO, Geneva.

[2] World Health Organization (2018) Global Health Workforce Statistics. World Health Organization, Geneva.

[3] World Health Organization (WHO) (2007) Task Shifting to Tackle Health Worker Shortages. WHO, Geneva.

[4] Ministry of Health (2015) Report of the Training Needs Assessment of Kenya's Health Workforce. http://www.health.go.ke

[5] MoH (2014) Human Resources for Health Norms and Standards Guidelines for the Health Sector-Kenya.

[6] GoK (2017) Task Sharing and Task Shifting Policy Guidelines (2017-2030).

[7] $\mathrm{MoH}$ (2014) Strategy for Community Health in Kenya-2014-2019.

[8] MoH (2019) Kenya Human Resources for Health Strategic Plan 2019-2023. 
[9] World Health Organization (2012) Optimizing Health Worker Roles to Improve Access to Key Maternal and Newborn Health Interventions through Task Shifting. World Health Organization, Geneva.

[10] MoH (2014) Kenya Health Policy 2014 to 2030.

[11] UNFPA (2014) Investing in Sexual and Reproductive Health in Sub Saharan Africa. Guttmacher Institute, New York.

[12] World Health Organization, PEPFAR \& UNAIDS. (2007)» Task Shifting: Rational Redistribution of Tasks among Health Workforce Teams: Global Recommendations and Guidelines. World Health Organization, Geneva. https://apps.who.int/iris/handle/10665/43821

[13] United Nations. Department of Economic and Social Affairs, Population Division. 2013 Update for the MDG Database: Unmet Need for Family Planning.

[14] UNFPA, Human Resources for Maternal Survival: Task Shifting to Non-Physician Clinicians, 2009 Addis Ababa United Nations Population Fund, United Nations Children's Fund, Averting Maternal Death and Disability Mailman School of Public Health University of Colombia, Ministry of Health Ethiopia.

[15] (2016) Kenya's Reproductive Maternal Neonatal Child and Adolescent Health (RMNCAH) Investment Framework for Kenya.

[16] Kenya Family Planning Costed Implementation Plan of 2017-2020.

[17] UN Population Division (2011) United Nations, World Population Prospects: The 2010 Revision. UN Population Division, New York.

[18] Kessler, I., Heron, P. and Dopson, S. (2012) The Modernization of the Nursing Workforce: Valuing the Healthcare Assistant. OUP, Oxford.

[19] Di Giorgio, L., et al. (2018) Cost of Administering Injectable Contraceptives through Health Workers and Self Injection: Evidence from Burkina Faso, Uganda and Senegal. Contraception, 98, 389-395.

https://doi.org/10.1016/j.contraception.2018.05.018

[20] Ali, M., Seuc, A., Rahimi, A., Festin, M. and Temmerman, M. (2014) A Global Research Agenda for Family Planning: Results of an Exercise for Setting Research Priorities. Bulletin of the World Health Organization, 92, 93-98. https://doi.org/10.2471/BLT.13.122242

[21] Aliyu, A. (2018) Family Planning Services in Africa: The Successes and Challenges.

[22] Lehmann, U., Van Damme, W., Barten, F. and Saunders, D. (2009) Task Shifting: The Answer to the Human Resources Crisis in Africa? Human Resources for Health, 7, Article No. 49. https://doi.org/10.1186/1478-4491-7-49

[23] Gueye, B., Wesson, J., Koumtingue, D., Stratton, S. and Viadro, C. (2016) Mentoring, Task Sharing, and Community Outreach through the TutoratPlus Approach: Increasing Use of Long-Acting Reversible Contraceptives in Senegal. Global Health: Science and Practice, 4, 33-43. https://doi.org/10.9745/GHSP-D-15-00328

[24] WHO (2018) WHO Guideline on Health Policy and System Support to Optimize Community Health Worker Programmes. https://apps.who.int/iris/bitstream/handle/10665/275474/9789241550369-eng.pdf?u $\underline{\mathrm{a}=1}$

[25] Westoff, C.F. (2006) New Estimates of Unmet Need and the Demand for Family Planning. DHS Comparative Reports, Macro International, Calverton.

[26] Bryce, J., et al. (2005) Programmatic Pathways to Child Survival: Results of a Multi-Country Evaluation of Integrated Management of Childhood Illness. Health Policy and Planning, 20, i5-i17. https://doi.org/10.1093/heapol/czi055 
[27] Briggs, C.J. and Garner, P. (2006) Strategies for Integrating Primary Health Services in Middle- and Low-Income Countries at the Point of Delivery. Cochrane Database of Systematic Reviews, No. 2, Article ID: CD003318.

https://doi.org/10.1002/14651858.CD003318.pub2

\section{List of Abbreviations}

CHDs: County Health Departments

CHWs: Community Health Workers

CPR: Contraceptive Prevalence Rate

CYP: Couple Years of Protection

FGDs: Focused Group Discussions

FP: Family Planning HRH: Human Resources for Health

KII: Key Informant Interviews

TSTSPG: Task Sharing and Task Shifting Policy Guidelines 